\title{
Reconfigurable and Tunable Filtenna for Cognitive LTE Femtocell Base Stations
}

\author{
Hamza Nachouane, Abdellah Najid, Abdelwahed Tribak, and Fatima Riouch
}

National Institute of Posts and Telecommunications (INPT), 10100 Rabat, Morocco

Correspondence should be addressed to Hamza Nachouane; nachouane@inpt.ac.ma

Received 15 March 2016; Revised 16 May 2016; Accepted 17 May 2016

Academic Editor: Chien-Jen Wang

Copyright ( 2016 Hamza Nachouane et al. This is an open access article distributed under the Creative Commons Attribution License, which permits unrestricted use, distribution, and reproduction in any medium, provided the original work is properly cited.

\begin{abstract}
A single-port uniplanar antenna with a built-in tunable filter is presented for operation in multiple LTE bands for cognitive femtocell applications. The antenna is based on a monopole microstrip patch fed by coplanar waveguide. The frequency reconfigurability is achieved by using two PIN diodes to couple or decouple a ring slot resonator filter from the antenna feed line. By switching the PIN diodes, the proposed design can operate in either wideband or narrowband modes. When the antenna operates in the narrowband state, two varactor diodes are used to continuously tune the narrowband frequency from 2.55 to $3.2 \mathrm{GHz}$, while the wideband state is obtained over the $1.35-6.2 \mathrm{GHz}$ band. The diodes and their biasing networks have nearly no severe effect on the antenna characteristics. Prototypes of the proposed structure using ideal and real switches, with and without varactors, are fabricated and tested. Measured and simulated results are in good agreement, thus verifying the good performance of the proposed design. The obtained results show that the proposed antenna is very suitable for cognitive radio applications, in which the wideband mode is used for spectrum sensing and the narrowband mode for transmission at different frequency bands.
\end{abstract}

\section{Introduction}

Recently, a "data tsunami" has resulted from the emergence of data-hungry wireless applications. Besides, the exponential growth in the number of smartphones and various portable devices has fueled the explosion of data traffic. In order to support higher throughput and better user satisfaction, the Long Term Evolution (LTE) standard has been proposed [1] Moreover, in the most recent surveys [2,3], it has been found that, in the near future, more than $60 \%$ of voice calls and $90 \%$ of data traffic will be originated from inside buildings. However, the traditional base stations have the reputation of poor indoor coverage due to the multipath and penetration losses $[4,5]$. Furthermore, the LTE system operates at higher frequencies when the attenuation is highly significant. This severely reduces the network capacity and coverage in dense areas. Hence, it is important to find an alternative solution to improve the coverage and data rate in indoor environments, especially at higher frequencies.

Femtocells are considered as a promising solution not only to overcome the indoor coverage issue but also to achieve higher data rates by shorting the communication range and a better frequency reuse capability $[6,7]$. Frequency reuse is a practical solution to overcome the spectrum scarcity [8], which is due principally to the increasing demand for more bandwidth versus the fact that all frequency bands are already allocated by the Federal Communications Commission (FCC). Another promising technology to meet the high data rate requirements and overcome the frequency scarcity is the cognitive radio (CR) paradigm [9]. It is able to continuously supervise the RF spectrum, in order to detect the spectrum holes, so-called white spaces, and, then, dynamically tune its characteristics to operate within these unused frequency bands [10]. By harnessing the advantages of these two technologies, cognitive femtocell base stations provide new opportunities for the next generation wireless networks, especially for LTE networks, to achieve high-quality service, maximize the efficiency of spectrum usage, and improve indoor coverage [11, 12].

The RF front-end of such systems must ensure their fundamental functions, which are the sensing and communication tasks [13]. Therefore, the reconfigurable antennas are 
TABLE 1: Comparison between the proposed filtenna and related works.

\begin{tabular}{|c|c|c|c|c|}
\hline Reference & $\begin{array}{l}\text { Narrowband frequency } \\
\text { tuning }(\mathrm{GHz})\end{array}$ & Wideband mode $(\mathrm{GHz})$ & $\begin{array}{c}\text { Number of } \\
\text { switches }\end{array}$ & Size $\left(\lambda_{g} \times \lambda_{g}\right)$ \\
\hline [15] & 3.9 to 4.82 & $3.8-6$ & $\begin{array}{l}1 \text { PIN diode } \\
2 \text { varactors }\end{array}$ & $1.24 \times 0.56$ \\
\hline [23] & $2.4 ; 4.2 ; 3.3 ; 5.4$ & $2-10$ & 2 FETs & $0.69 \times 0.69$ \\
\hline$[24]$ & $0.58 ; 0.86 ; 1.1 ; 2.48$ & $0.7-3.44(-6 \mathrm{~dB})$ & $\begin{array}{l}2 \text { PIN diodes } \\
2 \text { varactors }\end{array}$ & $0.49 \times 0.27$ \\
\hline [25] & $2.48 ; 3.5 ; 5.5 ; 2.45 ; 5.49$ & $2.95-10.92$ & 4 PIN diodes & $0.68 \times 0.68$ \\
\hline$[26]$ & $1.1 ; 2.25 ; 3.1$ & $1.0-3.2$ & 8 PIN diodes & $1.06 \times 1.04$ \\
\hline$[27]$ & $2.75 ; 2.86 ; 3.1 ; 3.26$ & $2.63-3.7$ & 12 PIN diodes & $1.22 \times 0.91$ \\
\hline Proposed filtenna & $2.55 ; 2.84 ; 3.05 ; 3.2$ & $1.35-6.2$ & $\begin{array}{l}2 \text { PIN diodes } \\
2 \text { varactors }\end{array}$ & $0.73 \times 0.54$ \\
\hline
\end{tabular}

the most suitable to meet these requirements [14], since they can ensure spectrum sensing by acting as an omnidirectional ultra-wideband (UWB) antenna and as a tunable narrowband antenna for communicating [15-17]. In order to reduce the hardware complexity and cost, research communities are working toward moving some components and integrating them into another to create a more compact and less expensive system. To this end, it is suggested to combine the frequency-selective filter and the antenna into a single structure so as to achieve a so-called filtering antenna or "filtenna." The filtenna is a planar antenna with a built-in bandpass filter, which can be used to provide frequency agility for communicating at different frequencies, without causing any interference to the adjacent bands and also to avoid undesired frequencies when it is used as a receiver. Despite many concurrent designs available for filtennas [15, 18-22], the reconfigurable filtennas are rarely reported.

In this paper, the design of a new reconfigurable and tunable filtenna, by using a tunable bandpass filter based on ring slot resonator (RSR), is proposed for cognitive femtocell base stations. UWB to narrowband reconfiguration is achieved by employing two PIN diodes that couple or decouple the RSR filter from the antenna feed line, thereby making the filtenna work either in sensing or communication modes. When the filtenna is in the communication mode, the narrowband frequency tunability is attained by integrating two varactor diodes into the RSR filter. The frequency tunability is achieved to make the proposed filtenna communicate at different frequency bands. The most distinguishing feature of the proposed filtenna can be summarized in the fact that the tunable filter, which is part of the antenna design, provides frequency and bandwidth reconfigurability without affecting the radiation characteristics of the antenna. Moreover, the filtenna has a simple topology which makes it easy to be scaled to higher or lower frequencies. In addition, compared to $[15,18-29]$, the proposed filtenna can achieve a continuous frequency tuning and UWB to narrowband reconfiguration by using only two PIN and two varactor diodes, thus simplifying and making a more flexible design. Measured results show a very wide impedance bandwidth from $1.3 \mathrm{GHz}$ to more than $6 \mathrm{GHz}$, which fully covers LTE bands $1 / 2 / 7 / 33$ 41 , while the narrowband frequency can realize a continuous frequency tuning from 2.55 to $3.2 \mathrm{GHz}$, covering the LTE bands $7 / 38 / 40 / 41$. To the best of the authors' knowledge, no femtocell-based cognitive single-port filtenna design using a simple filter and incorporating frequency reconfigurability and tunability has been published.

In Table 1, the performance of the proposed filtenna is compared with those of recently published papers. It is clearly observed that the proposed filtenna has more narrowband states and wide bandwidth for sensing state with only two PIN and varactor diodes than any of the reported designs. Moreover, the size of the proposed structure is smaller than the other designs based on their lower operating frequency.

\section{Ring Slot Resonator Filter Design}

In principle, the quarter-wavelength open-circuited stub presents a stopband response at the calculated frequency. Thus, by cascading two quarter-wavelength open-circuited stubs, calculated at two different frequencies, a bandpass filter can be obtained at the center frequency. A single ring slot produces a stopband response since it is acting as an opencircuited circuit at the calculated frequency. However, by cascading two of them, a passband response can be produced at the center frequency. Because of space constraints, a single ring slot can produce a passband response by bridging the slot at an appropriate location, which creates two short-circuited stubs.

To understand the ring slot mechanism, the simple and bridged slot configurations shown in Figures 1(a) and 1(b), respectively, were investigated. The simple ring slot is acting as a bandstop, when its average perimeter, $P_{s}=36 \mathrm{~mm}$, is equal to a half-wavelength at the stopped frequency $2.4 \mathrm{GHz}$, as shown in Figure 2(a). However, when the slot is bridged, as shown in Figure 2(b), two short-circuited stubs in series that have different lengths $P_{2}=26 \mathrm{~mm}$ and $P_{1}=10 \mathrm{~mm}$ are created, which represent a stopband response at the corresponding quart-wavelength at 1.5 and $4 \mathrm{GHz}$, respectively, as shown in Figure 2(b). Consequently, the bridged slot ring configuration produces a passband response at the center frequency $2.4 \mathrm{GHz}$.

\section{Sensing Antenna Design}

As aforementioned, the sensing task is ensured by a UWB omnidirectional antenna. The initial design was started with the configuration shown in Figure 3. 

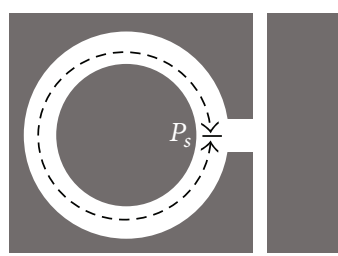

(a)

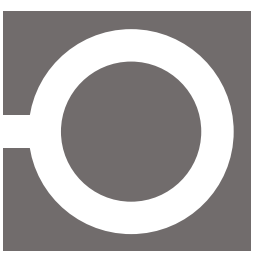

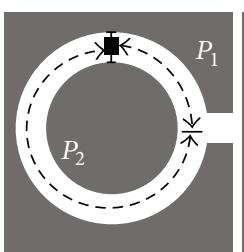

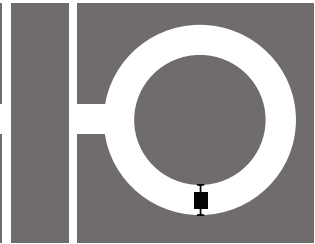

(b)

FIGURE 1: Configuration of a single bandstop (a) and (b) bandpass filters.

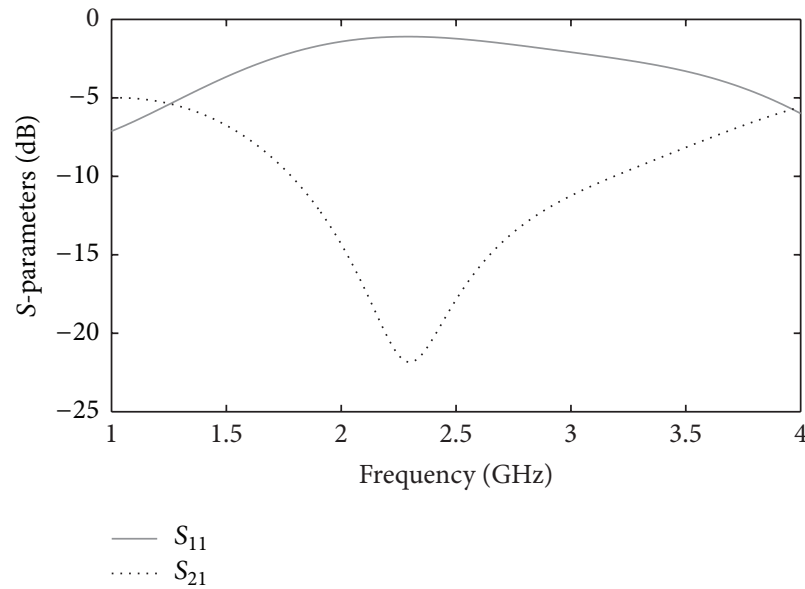

(a)

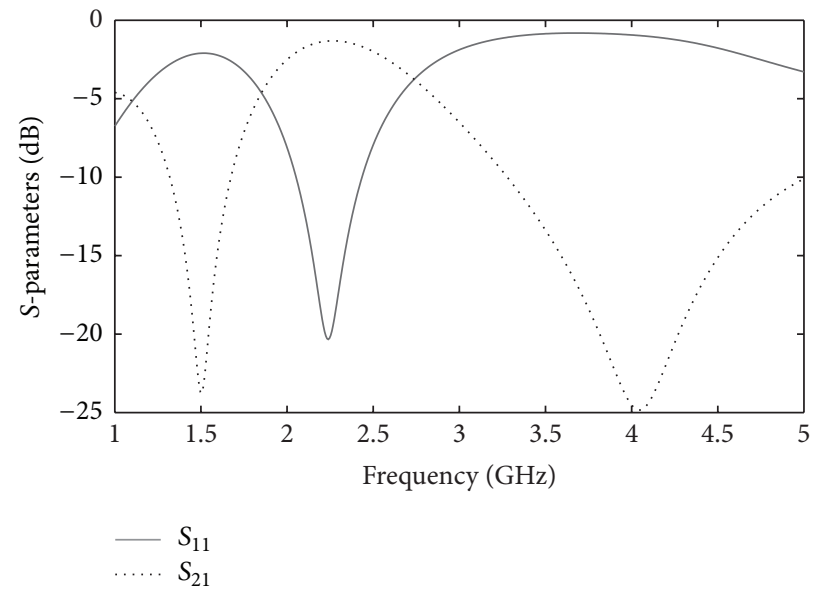

(b)

FIGURE 2: $S$-parameters of (a) the simple and (b) the bridged ring slot configurations.

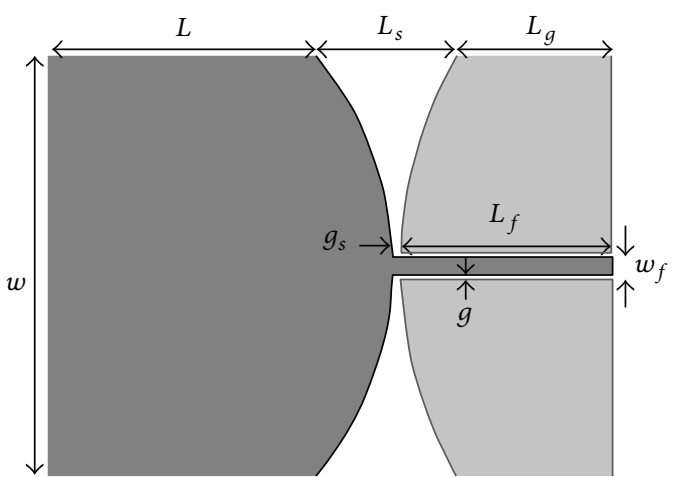

FIgURe 3: Configuration of the basic UWB antenna.

The basic structure of the proposed filtenna is based on a wideband monopole antenna operating from 1.33 to $6.5 \mathrm{GHz}$, based on $10 \mathrm{~dB}$ return loss level, which is $132 \%$ around the center frequency $3.9 \mathrm{GHz}$. It consists of a monopole antenna fed by CPW line and designed on the same side of the substrate as the ground plane. A $1.58 \mathrm{~mm}$ thick FR4 substrate, with dielectric constant of 4.3 and $\tan \delta=0.02$, is used in this work. The dimensions of the basic UWB antenna and the CPW line are listed in Table 2, which are calculated using equations provided in $[28,29]$. The photograph of the fabricated UWB antenna prototype as well as the simulated and measured return loss results are illustrated in Figure 4.
TABLe 2: Dimensions of the basic antenna.

\begin{tabular}{lcccccccc}
\hline Parameters & $L$ & $w$ & $L_{s}$ & $L_{g}$ & $L_{f}$ & $g$ & $g_{s}$ & $w_{f}$ \\
\hline Value $(\mathrm{mm})$ & 38 & 60 & 20 & 22 & 30 & 0.5 & 1.5 & 3 \\
\hline
\end{tabular}

To choose the appropriate location to integrate the filter into UWB sensing antenna, the surface current distribution on the UWB antenna at $4 \mathrm{GHz}$ is simulated, as shown in Figure 5. It can be clearly observed that the edges of the CPW line and the tapered slots have high current density. This suggests that this region is acting as a transmission part while the rest of monopole antenna is acting as a radiating part. The transmission region can thus be effectively utilized to integrate the RSR filter for an efficient filtering. The basic topologies of the RSR filter and the UWB antenna are now described.

\section{Filtenna Fabrication and Measurements}

4.1. Wide to Narrow Bandwidth Reconfiguration. After designing the UWB sensing antenna and choosing the final configuration of the RSR filter providing a bandpass response at $2.4 \mathrm{GHz}$, both structures are combined in a single structure to get the final filtenna, as shown in Figure 6(a). To examine the effect of the RSR filter on the antenna, an ideal switch is used. The OFF state is indicated by a gap while the $\mathrm{ON}$ state is represented by a metal pad. The aim of using ideal 


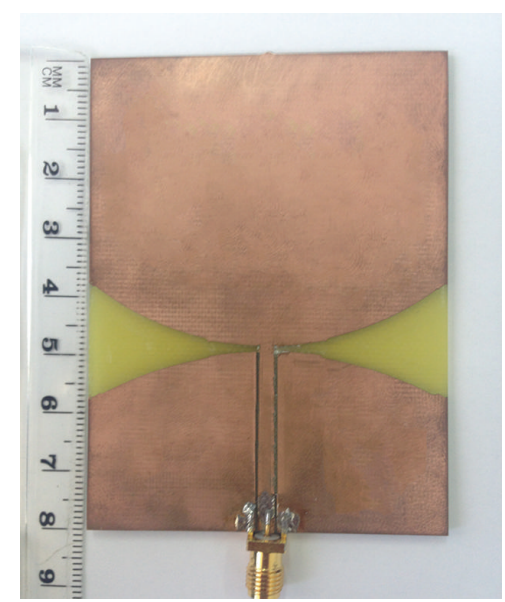

(a)

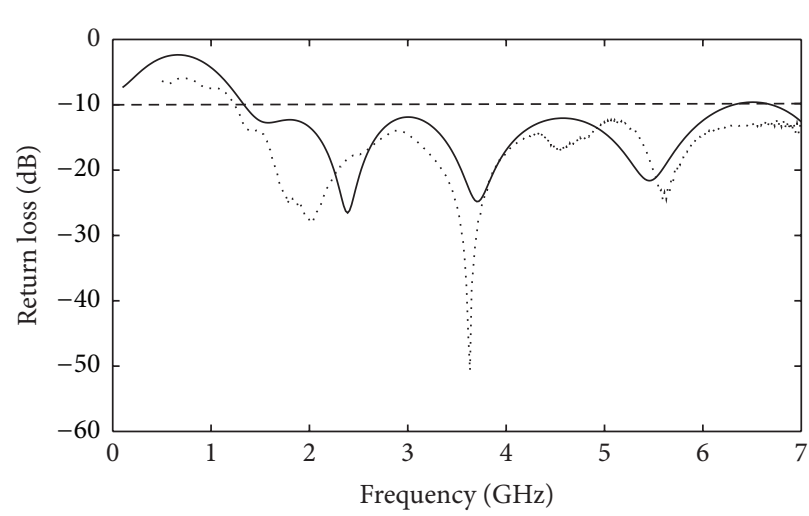

Measured

— Simulated

FIGURE 4: (a) Photograph of the fabricated UBW antenna and (b) simulated and measured return loss.

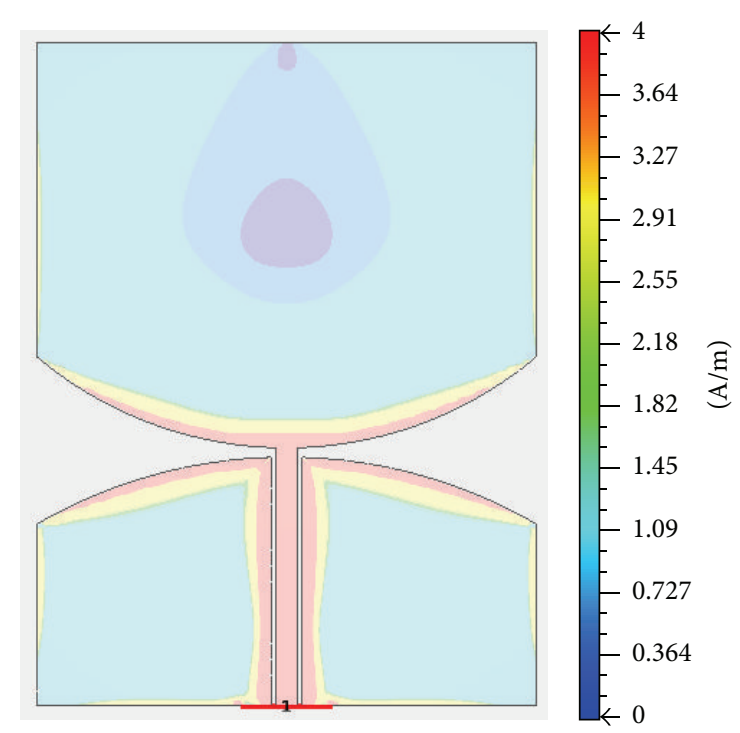

FIGURE 5: Simulated surface current distribution at $4 \mathrm{GHz}$.

switches, in the first place, is to show the effect of active components (PIN diodes and their biasing networks) on the antenna characteristics. The RSR filter is located in the middle of the ground plane to avoid both the SMA connector and the strong coupling from the radiating part.

Figure 6(b) shows the antenna behavior when the filter is coupled and decoupled. As it can be noticed, the bandwidth reconfigurability is achieved. When the filter is decoupled from the antenna feed line (ON state), the filtenna acts as a UWB sensing antenna. The measured $10 \mathrm{~dB}$ impedance bandwidth covers the range from 1.3 to $6.6 \mathrm{GHz}$, which is $134 \%$ around the center frequency $3.95 \mathrm{GHz}$. However, when the filter is coupled to the antenna feed line through the gaps (OFF state), the filtenna carries out the communication task at $2.4 \mathrm{GHz}$, by switching from wide to narrow bandwidth. To further understand the filter mechanism, the surface current distributions for the narrowband mode are simulated at the operating frequency $2.4 \mathrm{GHz}$ and at out-of-band frequencies 1 and $4 \mathrm{GHz}$, as shown in Figure 7. It is observed that the RSR filter passes the current at $2.4 \mathrm{GHz}$ while reducing the current density on the antenna radiating element at the other frequencies.

The ideal switches reduce the computation time and have low insertion loss, but they do not provide fast and smooth switching, which are highly required in CR systems. Therefore, a real switch based on PIN diode is used instead. In this work, Infineon PIN diodes, model BAR88-02V, are used. According to their data sheet [30], for simulation purposes, the diode can be modeled as a $2.5 \Omega$ resistor for the ON state and a parallel circuit of a $0.4 \mathrm{pF}$ capacitor and a $65 \mathrm{k} \Omega$ resistor for the OFF state, as shown in Figure 8. Two PIN diodes are implemented in each gap of the RSR filter to decouple and couple it to the antenna. For experimental purposes, a gap of $0.8 \mathrm{~mm}$ is created to prevent the DC current from flowing directly to the ground plane. However, the RF continuity is maintained by bridging the gap with $22 \mathrm{pF}$ surface mount device (SMD) capacitors. In order to protect the DC source from the RF signal, two high-impedance DC lines were printed on either side of the filtenna and connected to the ground plane via $33 \mathrm{nH}$ SMD inductors, as shown in Figure 9.

Based on the aforementioned analysis, a prototype of the proposed filtenna is fabricated using LPKF milling machine to validate the circuit concept, as shown in Figure 10(a). The measurements were performed using an Anritsu MS2028C Vector Network. The simulated and measured return loss results for both states are illustrated in Figure 10(b). UWB sensing operation is achieved over the $1.25-6.2 \mathrm{GHz}$ band, with good agreement observed between the simulated and measured results. The narrowband response is obtained at $2.45 \mathrm{GHz}$, corresponding to LTE band 38 , with a slight discrepancy between the two curves, especially at higher frequencies. This can be understood by considering the high insertion loss of diodes as the frequency is increased. Besides, the manufacturing tolerances and the parasitic associated 


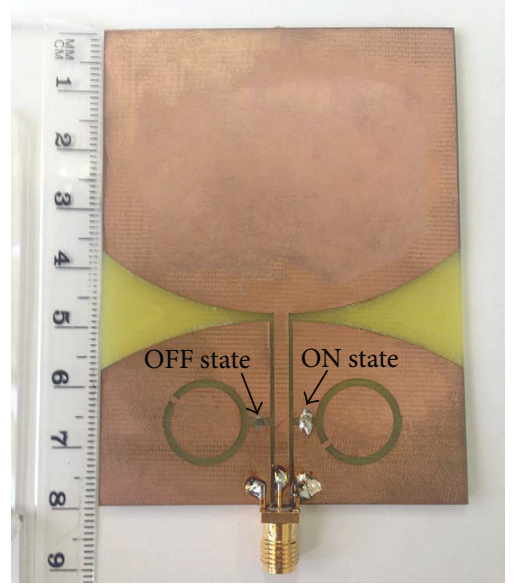

(a)

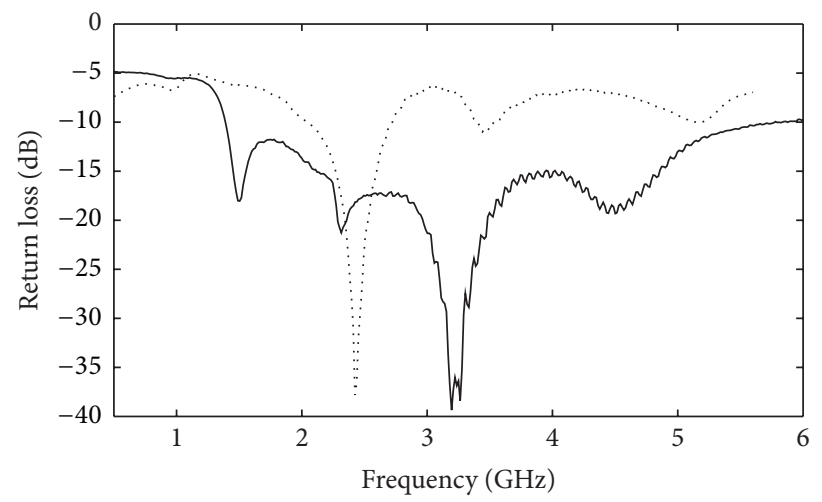

OFF state

ON state

FIgURE 6: (a) Photograph of the fabricated structure and (b) measured return loss for each state using ideal switches.

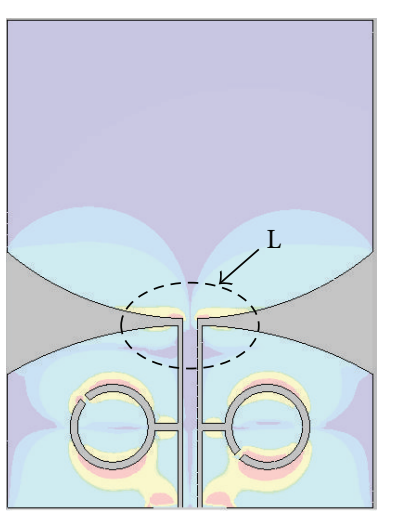

(a)

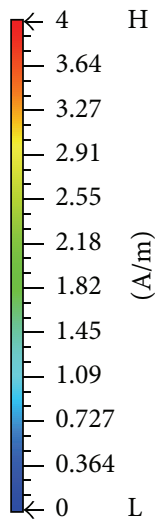

$\mathrm{L}$

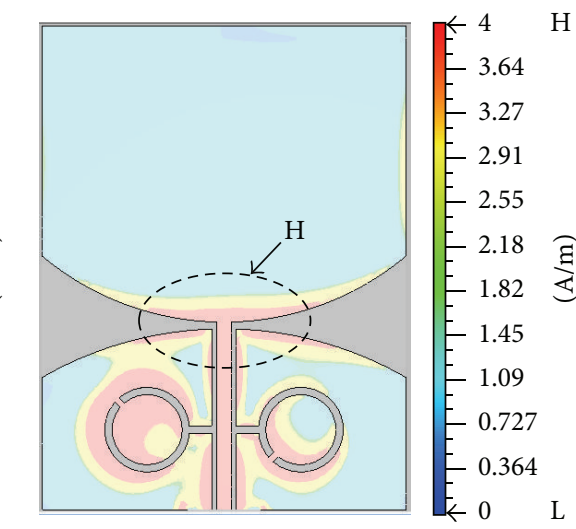

(b)

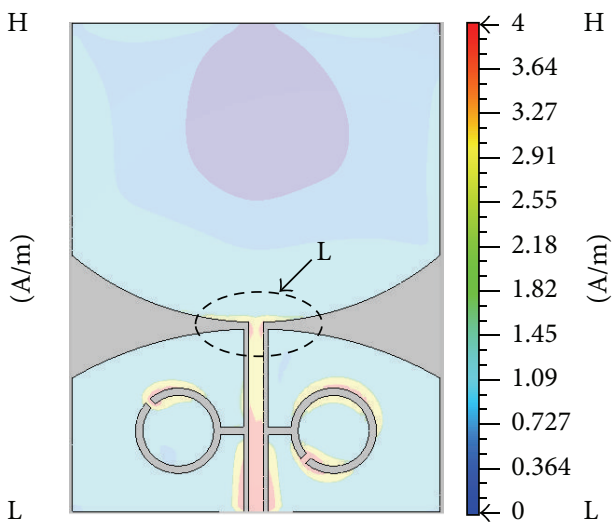

(c)

Figure 7: Surface current distributions at (a) $1 \mathrm{GHz}$, (b) $2.4 \mathrm{GHz}$, and (c) $4 \mathrm{GHz}$.

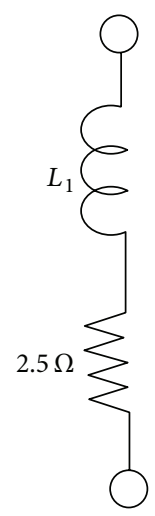

(a)

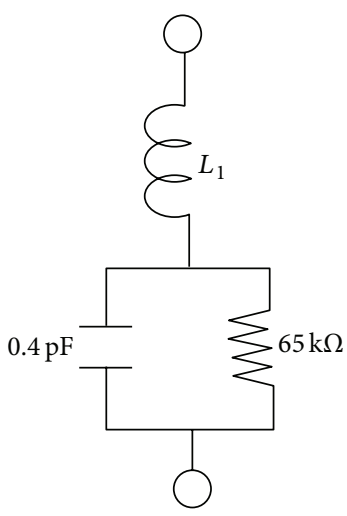

(b)
FIGURE 8: Equivalent circuits of PIN diode in (a) ON state and (b) OFF state.

with the diode package and bias components may considerably contribute to the frequency shifting. Furthermore, it is

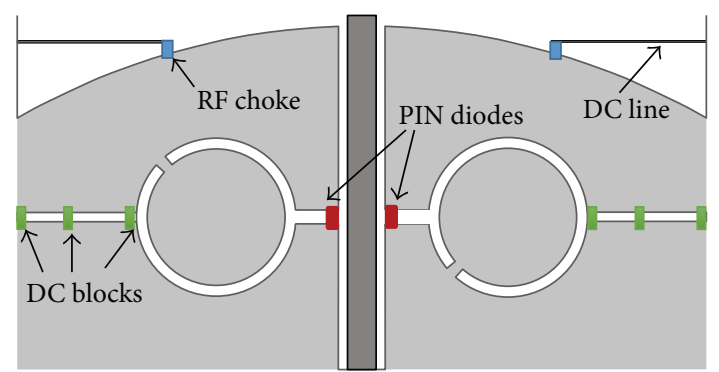

FIGURE 9: Schematic of the proposed biasing technique.

observed that, by using real switches, the measured resonance is shifted from 2.43 to $2.45 \mathrm{GHz}$ compared to ideal switches.

Moreover, to analyze the effect of PIN diodes on the antenna gain, a comparison between the ideal and real switches configurations is made in Table 3. It can be clearly observed that the measured gain in sensing mode is lower than in the communication mode. This result is principally attributed to the nature of the communication operation in 


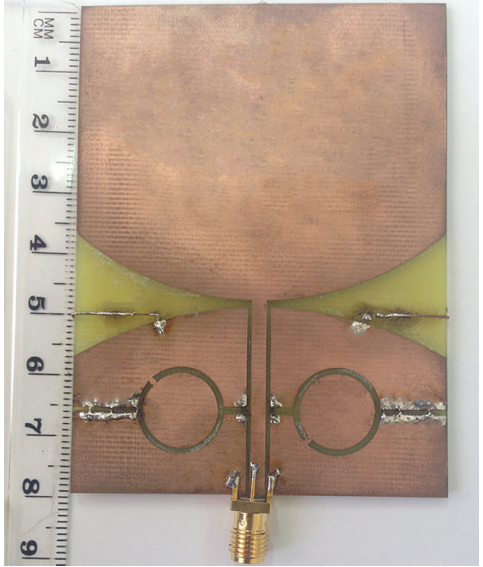

(a)

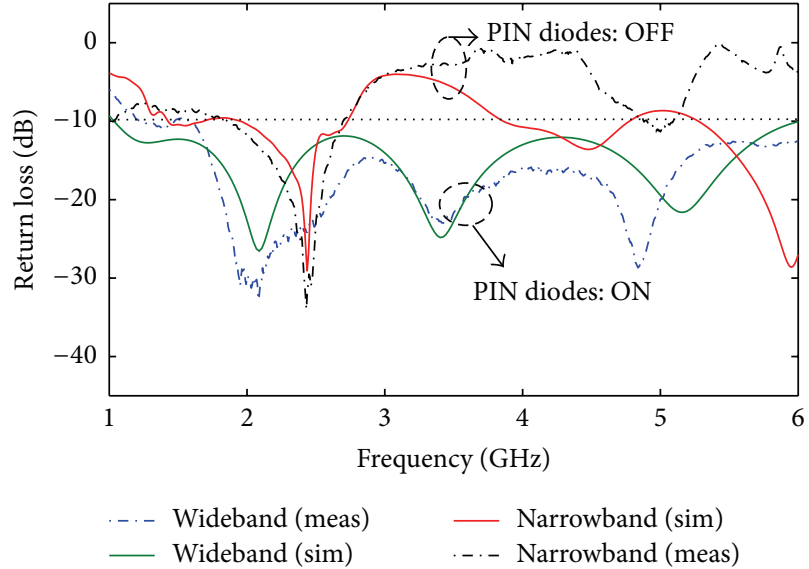

(b)

Figure 10: (a) Photograph of the fabricated filtenna and (b) simulated and measured return losses for each state.

TABLE 3: Measured gain of the proposed filtenna in both modes.

\begin{tabular}{lcccc}
\hline & \multicolumn{2}{c}{$\begin{array}{c}\text { Wideband mode } \\
\text { (at } 2.4 \mathrm{GHz})\end{array}$} & \multicolumn{2}{c}{ Narrowband mode } \\
(at 2.45 GHz) \\
Ideal & Real & Ideal & Real \\
switches & switches & switches & switches \\
\hline Gain, dBi & 3.9 & 3.55 & 6.61 & 5.12 \\
\hline
\end{tabular}

providing a high gain on the signal-of-interest for maintaining a robust link between users in CR networks. On the other hand, the structure with ideal switches has up to $1.49 \mathrm{~dB}$ higher gain than the one with the real switches. This can be explained by the loss of the PIN diode switches and their biasing components, which is related to their internal resistances.

4.2. Narrowband Frequency Tunability. As previously mentioned, the communication task is achieved at one frequency. To achieve the frequency tunability of the narrowband operation, the bridges are replaced by varactor diodes. The varactor diode is placed in the bridge position to vary the ring slot effective length and thereby effectively tune the passband center frequency. Two BB145 varactor diodes from NXP Semiconductors [31], whose capacitance can be tuned in the range of 2.55-7.2 $\mathrm{pF}$ for a corresponding supply voltage of 4 to $1 \mathrm{~V}$, are placed in each ring slot at the bridge location, as shown in Figure 11. An equivalent circuit used to model the diode in simulations is illustrated in Figure 12. Since the structure contains PIN and varactor diodes, their biasing circuits must thus be properly designed and separated to not negatively affect each other. The varactors biasing network is similar to that of the PIN diodes, with DC blocks of $22 \mathrm{pF}$ and RF chokes of $33 \mathrm{nH}$. In addition, two RF chokes are introduced in each slot to preserve the DC current continuity to ground, as illustrated in Figure 11. The photograph of the final prototype is depicted in Figure 13(a). A GW Instek AFG-2112 DC power

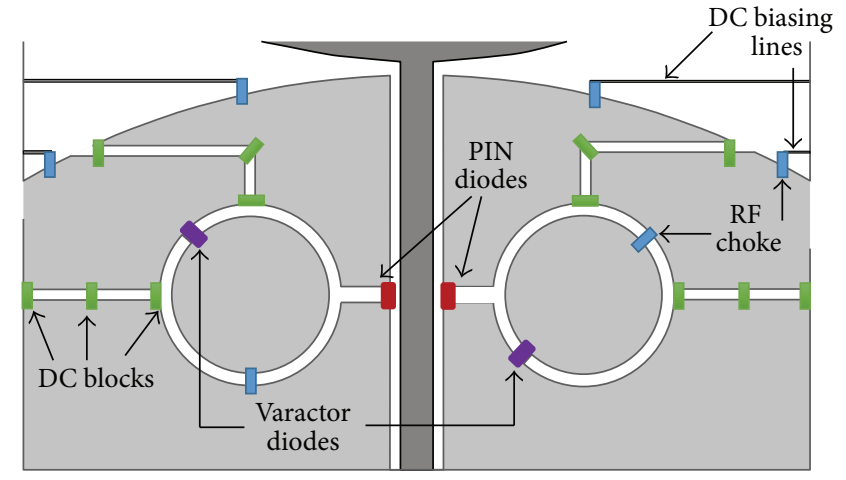

FIGURE 11: Diagram of the proposed DC supply.

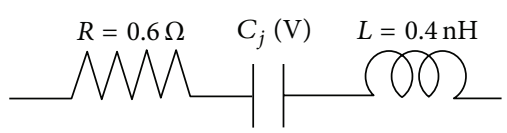

FIGURE 12: Equivalent circuit of the varactor diode, with $C_{j}=2.55-$ $7.2 \mathrm{pF}$.

supply unit is used to provide constant and variable DC bias voltage for PIN and varactor diodes, respectively.

The simulated and measured return loss results for different varactor capacitance values are given in Figures 13(b) and 13(c), respectively. It is observed that, by varying the varactor voltages from 1 to $4 \mathrm{~V}$ (i.e., by decreasing the capacitance value from 7.2 to $2.5 \mathrm{pF}$ ), the operating frequency can be continuously tuned from 2.55 to $3.2 \mathrm{GHz}$, corresponding to a 1.26 tuning ratio. These bands include LTE bands 7 $(2.5-2.57 \mathrm{GHz}), 38(2.57-2.62 \mathrm{GHz}), 40(2.3-2.4 \mathrm{GHz})$, and $41(2.496-2.69 \mathrm{GHz})$, while the simulated tuning range is from 2.6 to $3.4 \mathrm{GHz}$, with a reasonably good agreement with experimental results. The slight discrepancies between experimental and simulation results would be mainly attributed to the inaccuracies of manufacturing, FR4 relative permittivity, 


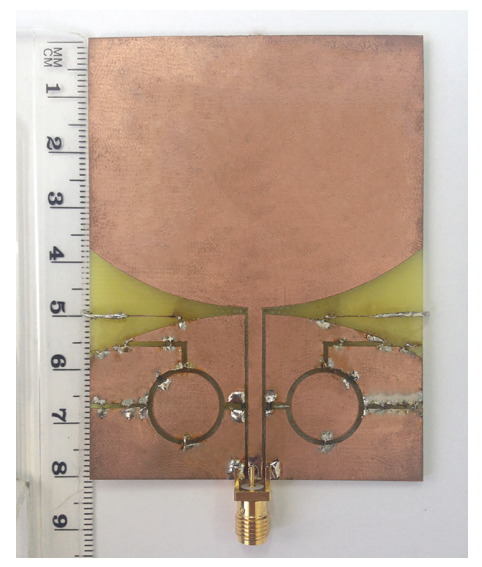

(a)

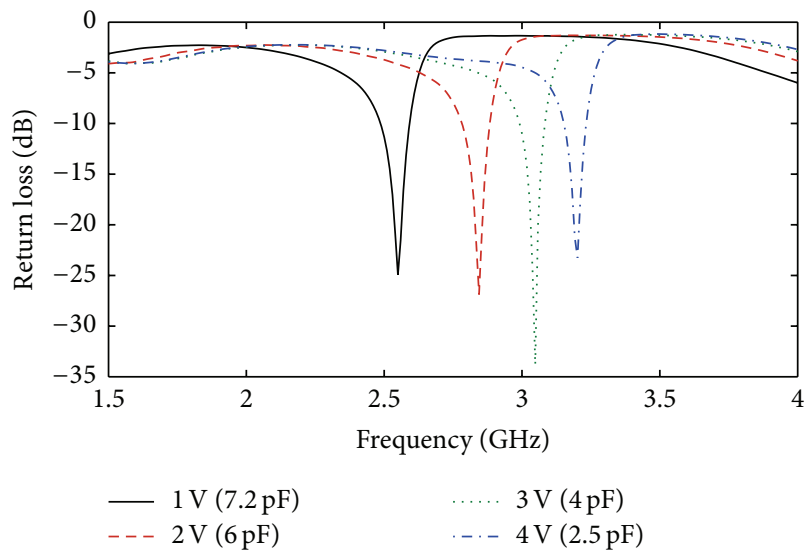

(b)

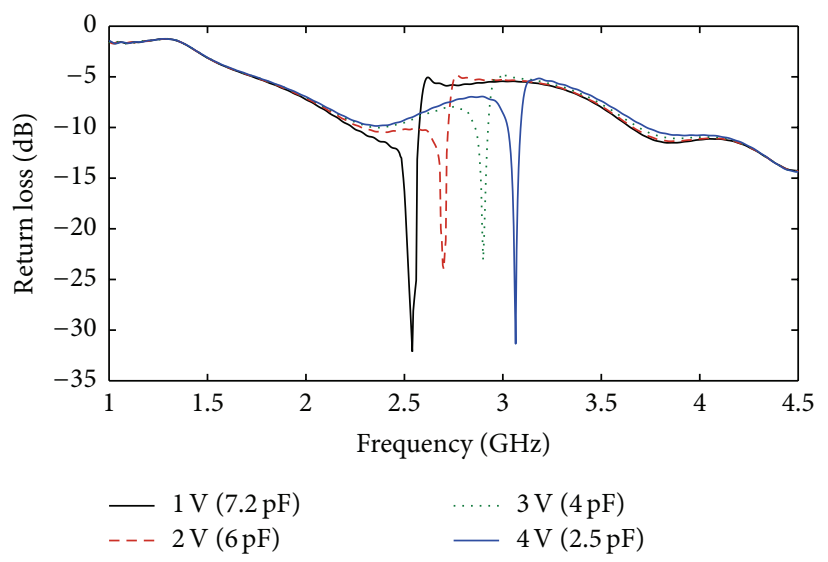

(c)

FIGURE 13: (a) Photograph of the final fabricated filtenna and its (b) simulated and (c) measured return losses in the narrowband mode for different varactor voltages.

bias voltage control error, and the lack of proper modeling of the discrete components in simulations. Moreover, a slight error can be introduced during the soldering of the SMD components and SMA connector which is not considered in simulations.

As explained earlier, the varactors are mainly used to tune the narrowband frequency, but their impact on sensing operation must be taken into consideration. The measured return losses for two varactor capacitance values, when the PIN diodes are set to ON, are plotted in Figure 14. It can be seen that the return loss remains nearly unchanged over the UWB sensing impedance bandwidth. Nevertheless, a slight variation can be observed at higher frequencies, but this change is negligible since the $10-\mathrm{dB}$ impedance bandwidth remains unchanged. This can be explained by the fact that the variation of the capacitance value on the ground plane results in the change of the effective electrical length of the antenna and thereby slightly shifted its operating bandwidth. The effects of changing the varactor capacitance on the gain are investigated as well, in order to make sure that tuning frequency does not affect the radiation characteristics of the proposed filtenna. The realized gain is compared for different varactor capacitance values, when the filtenna works in communication mode (when PIN diodes are OFF), as shown in Figure 15. It can be observed that the gain varies from 2.2 to $5.6 \mathrm{dBi}$, with a maximum gain at the operating frequency of each biasing voltage. The effect of the RSR filter is clearly observed in the gain variations, which indicates that the filtenna when operating in the communication mode is filtering the unwanted frequencies. However, the varactor diodes have nearly no severe impact neither on the sensing characteristics nor on the realized gain of the filtenna.

The radiation efficiency of the prototype in both modes is also measured using the Wheeler cap method [32] and compared in Figure 16. This method is very simple conceptually. It requires one measurement of the impedance with the antenna in free space and another where the antenna is enclosed with a small metallic cap placed at a distance greater than $\lambda / 2 \pi$ [33]. The real part of the first impedance measurement is equivalent to the loss resistance of the antenna $R_{r}$, while the real part of the second measurement includes the radiation resistance $R_{L}$ and loss resistance $R_{r}$. By using (1), the radiation efficiency can be calculated. From Figure 16, it is seen that the radiation efficiency is found to be better than $69 \%$ in 


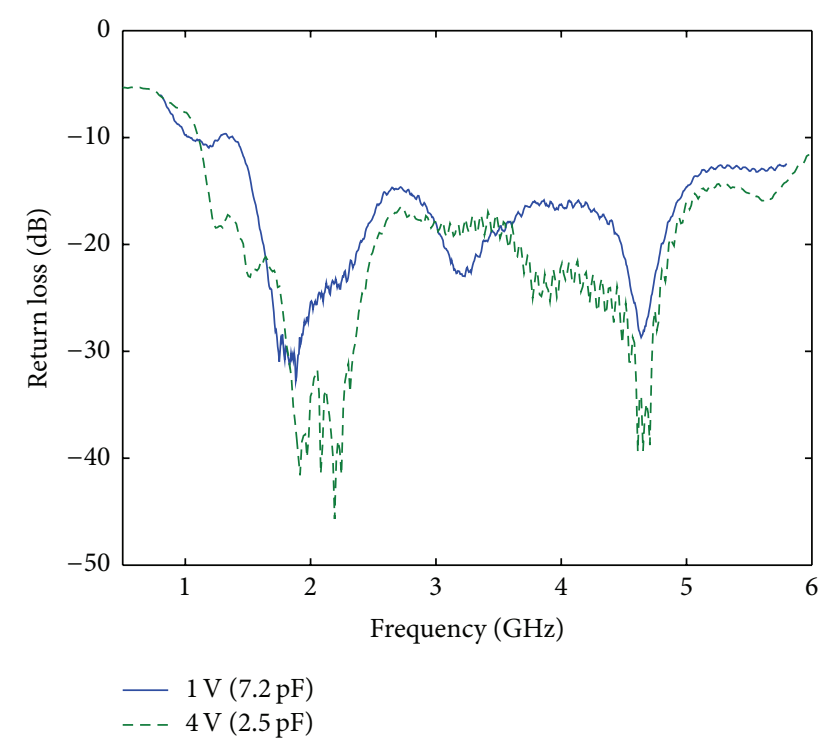

Figure 14: Measured return loss of the proposed filtenna in the wideband mode for two varactor voltages.

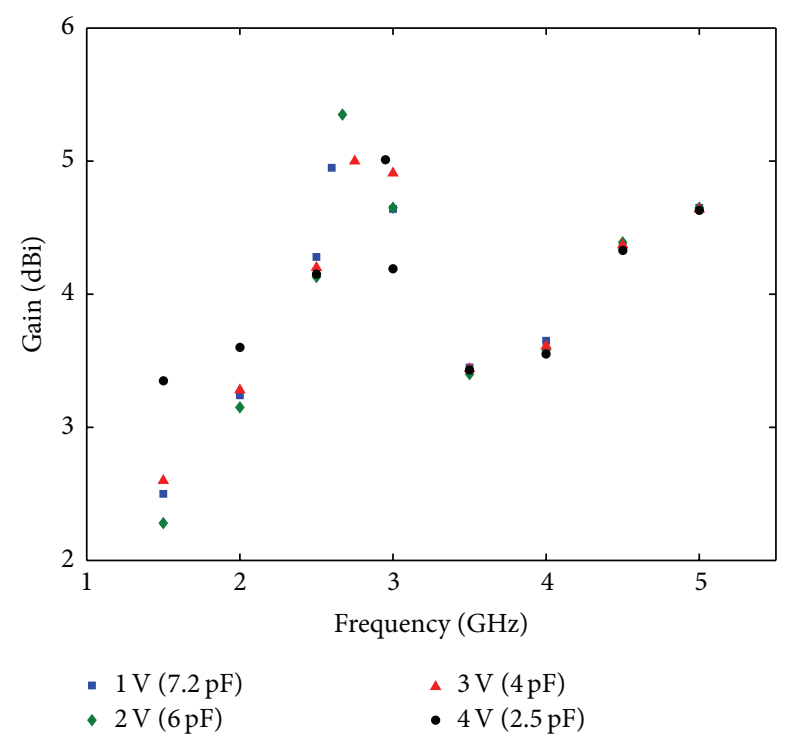

FIGURE 15: Gain of the proposed filtenna in the narrowband mode for different varactor voltages.

the wideband mode. In the narrowband mode, the efficiency is found to be better than $79 \%$. The difference between the two curves is because of the forward resistance of the PIN diodes when they are on which contribute to thermal losses:

$$
\eta_{e}=\frac{P_{t}}{P_{\text {in }}}=\frac{R_{r}}{R_{L}+R_{r}} .
$$

The radiation patterns of the proposed filtenna for both modes are shown in Figure 17. For the sensing state, the patterns are plotted at $1.5,3.5$, and $5 \mathrm{GHz}$, while for the narrowband state, they are plotted at the resonant frequency of the communication mode. It can be noticed that the

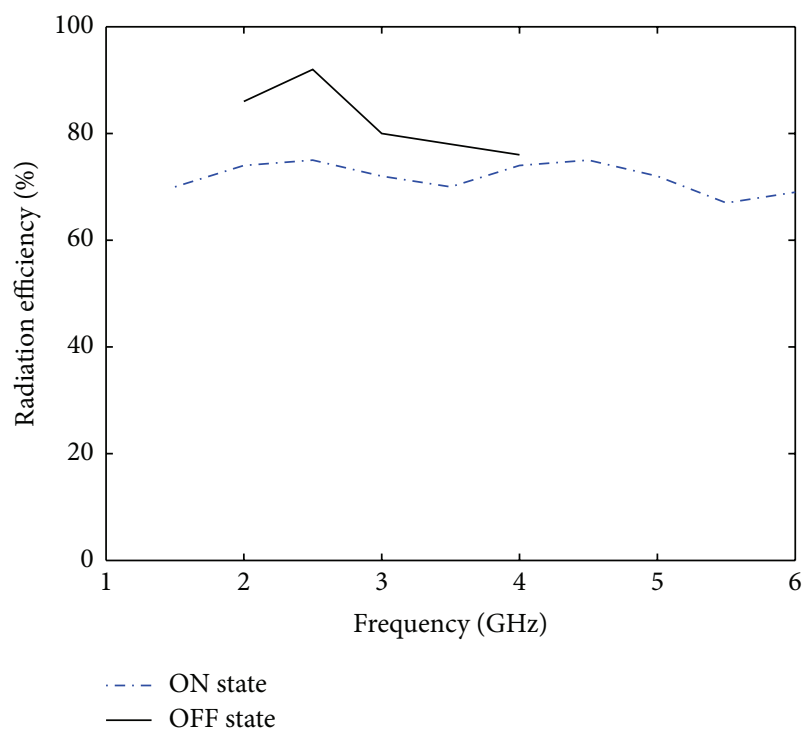

FIGURE 16: Measured radiation efficiency of the filtenna in wideband mode $(\mathrm{ON})$ and narrowband mode (OFF).

radiation patterns of sensing mode are slightly omnidirectional throughout the operating bandwidth with low crosspolarization level, which is better than $20 \mathrm{~dB}$ over the main beam direction. However, it can also be seen that the narrowband mode exhibits a quasi-doughnut shape with slightly lower cross-polarization levels, especially in the $Y-Z$ plane. This is mainly because, at higher frequencies, the effective electrical length of the ground plane is much longer than one wavelength, which leads to splitting the radiation pattern.

\section{Conclusion}

An electronically reconfigurable and tunable filtenna for cognitive femtocell base stations has been presented. The basic design of the filtenna consists of monopole antenna fed by CPW with a built-in RSR filter in the ground plane. To switch the filtenna between the sensing mode and the communication mode, two PIN diodes are used to couple or decouple the RSR filter from the antenna feed line. However, in order to continuously tune the resonant frequency of the communication mode, two varactor diodes are used to change the effective electrical length of the filter slots. Four filtenna prototypes have been manufactured and tested to verify the design concept. Each prototype could be regarded as an evolution of the previous prototype. Measured results have shown that the proposed filtenna provides an impedance bandwidth from 1.35 to $6.2 \mathrm{GHz}$ and 2.33 to $3.1 \mathrm{GHz}$ in sensing and communication modes, respectively, given a very wide bandwidth tuning ratio of $6.3: 1$. Moreover, the resonant frequency of the communication mode can be continuously tuned from 2.55 to $3.2 \mathrm{GHz}$, by varying the DC bias voltage from 1 to $4 \mathrm{~V}$, respectively, corresponding to a 1.26 frequency tuning ratio. Therefore the proposed design is expected to fully cover the LTE bands $1 / 2 / 7 / 33-41$. In addition, the gain, the radiation efficiency, and the radiation patterns show that the frequency tuning and reconfiguration 

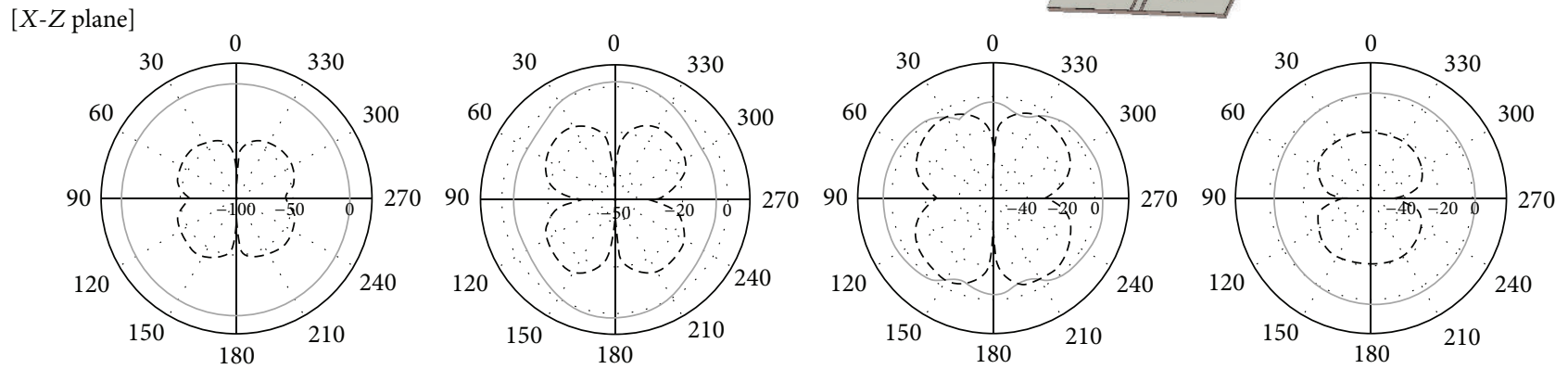

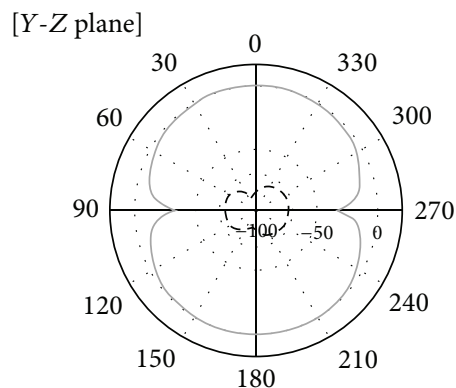

$1.5 \mathrm{GHz}$

[wideband mode]

- Co-polar

-- - Cx-polar
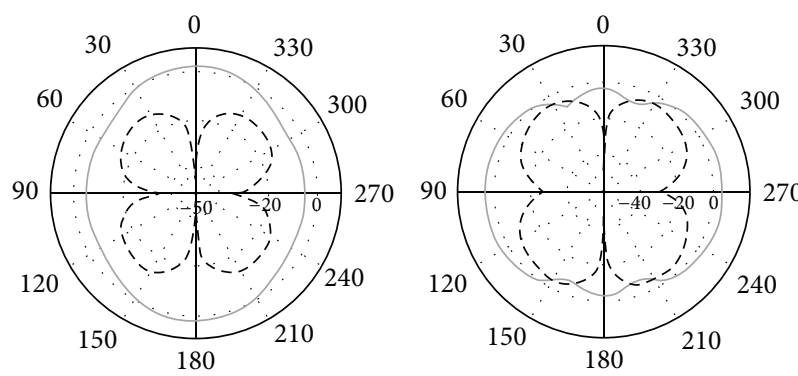

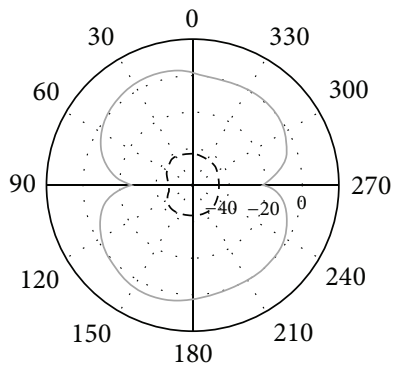

$3.5 \mathrm{GHz}$

[wideband mode]

Co-polar

- - - Cx-polar

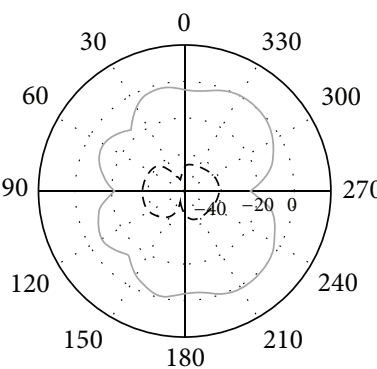

$5 \mathrm{GHz}$

[wideband mode]

Co-polar

- - Cx-polar

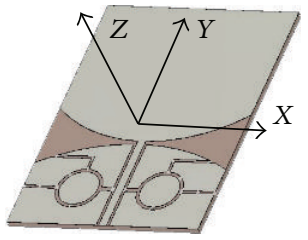

FIGURE 17: Radiation patterns of the proposed filtenna.

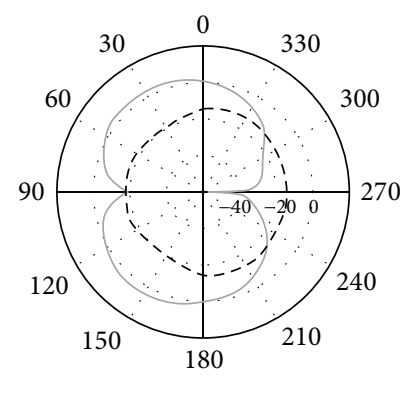

$2.5 \mathrm{GHz}$

[narrowband mode]

- Co-polar
-- Cx-polar mechanisms are accomplished without affecting the filtenna characteristics, qualifying the proposed design for use in many CR applications.

\section{Competing Interests}

The authors declare that there is no conflict of interests regarding the publication of this paper.

\section{Acknowledgments}

The authors are grateful to Departamento de Ingenieria de Comunicaciones, University of Cantabria (UNICAN), Spain, for support with regard to simulation software and facilities.

\section{References}

[1] 3GPP. TR 36.814, "Further advancements for E-UTRA physical layer aspects, v.9.0.0.," Tech. Rep. 3GPP, 2010.

[2] P. Lin, J. Zhang, Y. Chen, and Q. Zhang, "Macro-femto heterogeneous network deployment and management: from business models to technical solutions," IEEE Wireless Communications, vol. 18, no. 30, pp. 64-70, 2011.
[3] G. De la Roche and B. Allen, LTE-Advanced and Next Generation Wireless Networks: Channel Modelling and Propagation, John Wiley \& Sons, Chicago, Ill, USA, 2013.

[4] A. M. D. Turkmani, J. D. Parsons, and D. G. Lewis, "Measurement of building penetration loss on radio signals at 441, 900 and $1400 \mathrm{MHz}$,' Journal of the Institution of Electronic and Radio Engineers, vol. 58, no. 6, pp. S169-S174, 1988.

[5] E. H. Walker, "Penetration of radio signals into buildings in the cellular radio environment," The Bell System Technical Journal, vol. 62, no. 9, pp. 2719-2734, 1983.

[6] H. Claussen, L. T. W. Ho, and L. G. Samuel, "An overview of the femtocell concept," Bell Labs Technical Journal, vol. 13, no. 1, pp. 221-246, 2008.

[7] V. Chandrasekhar, J. G. Andrews, and A. Gatherer, "Femtocell networks: a survey," IEEE Communications Magazine, vol. 46, no. 9, pp. 59-67, 2008.

[8] V. Venkatkumar, T. Wirth, T. Haustein, and E. Schulz, "Relaying in long term evolution: indoor full frequency reuse," in Proceedings of the European Wireless Conference (EW'09), pp. 298-302, Aarlborg, Denmark, May 2009.

[9] J. Mitola and G. Q. Maguire, "Cognitive radio: making software radios more personal," IEEE Personal Communications, vol. 6, no. 4, pp. 13-18, 1999. 
[10] J. Mitola, Cognitive radio an integrated agent architecture for software defined radio [Ph.D. thesis], Royal Institute of Technology (KTH), Stockholm, Sweden, 2000.

[11] S. Bayhan, G. Gür, and F. Alagoz, "Cognitive capabilities for femtocell networks: cognitive femtocells," in Cognitive Radio and Its Application for Next Generation Cellular and Wireless Networks, V. Hrishikesh and G.-M. Muntean, Eds., vol. 116 of Lecture Notes in Electrical Engineering, pp. 331-356, Springer, 2012.

[12] P. Kulkarni, W. H. Chin, and T. Farnham, "Radio resource management considerations for LTE Femto cells," ACM SIGCOMM Computer Communication Review, vol. 40, no. 1, pp. 26-30, 2010.

[13] P. S. Hall, P. Gardner, and A. Faraone, "Antenna requirements for software defined and cognitive radios," Proceedings of the IEEE, vol. 100, no. 7, pp. 2262-2270, 2012.

[14] J. T. Bernhard, Reconfigurable Antennas, Morgan \& Claypool, San Rafael, Calif, USA, 2007.

[15] P.-Y. Qin, F. Wei, and Y. J. Guo, "A wideband-to-narrowband tunable antenna using a reconfigurable filter," IEEE Transactions on Antennas and Propagation, vol. 63, no. 5, pp. 2282-2285, 2015.

[16] M. Al-Husseini, K. Y. Kabalan, A. El-Hajj, and C. G. Christodoulou, "Reconfigurable microstrip antennas for cognitive radio," in Advancement in Microstrip Antennas with Recent Applications, A. Kishk, Ed., InTech, 2013.

[17] H. Nachouane, A. Najid, F. Riouch, and A. Tribak, "Two-port antenna for cognitive radio front-ends," International Journal of Microwave and Optical Technology, vol. 11, no. 1, 2016.

[18] G. Q. Luo, W. Hong, H. J. Tang et al., "Filtenna consisting of horn antenna and substrate integrated waveguide cavity FSS," IEEE Transactions on Antennas and Propagation, vol. 55, no. 1 , pp. 92-98, 2007.

[19] C. Yu, W. Hong, Z. Kuai, and H. Wang, "Ku-band linearly polarized omnidirectional planar filtenna," IEEE Antennas and Wireless Propagation Letters, vol. 11, pp. 310-313, 2012.

[20] C.-K. Lin and S.-J. Chung, "A filtering microstrip antenna array," IEEE Transactions on Microwave Theory and Techniques, vol. 59, no. 11, pp. 2856-2863, 2011.

[21] C.-T. Chuang and S.-J. Chung, "Synthesis and design of a new printed filtering antenna," IEEE Transactions on Antennas and Propagation, vol. 59, no. 3, pp. 1036-1042, 2011.

[22] Y. Yang and M. J. Lancaster, "Waveguide slot antenna with integrated filters," Proceedings of the 32nd European Space Agency Workshop, 2010.

[23] T. Aboufoul, A. Alomainy, and C. Parini, "Reconfiguring UWB monopole antenna for cognitive radio applications using GaAs FET switches," IEEE Antennas and Wireless Propagation Letters, vol. 11, pp. 392-394, 2012.

[24] D. Zhao, Y. Han, F. Liang, Q. Zhang, and B.-Z. Wang, "Lowpower optically controlled patch antenna of reconfigurable beams," International Journal of Antennas and Propagation, vol. 2014, Article ID 978258, 6 pages, 2014.

[25] H. Boudaghi, M. Azarmanesh, and M. Mehranpour, "A frequency-reconfigurable monopole antenna using switchable slotted ground structure," IEEE Antennas and Wireless Propagation Letters, vol. 11, pp. 655-658, 2012.

[26] M. R. Hamid, P. Gardner, P. S. Hall, and F. Ghanem, "Switchedband Vivaldi antenna," IEEE Transactions on Antennas and Propagation, vol. 59, no. 5, pp. 1472-1480, 2011.

[27] A. Mansoul, F. Ghanem, M. R. Hamid, and M. Trabelsi, "A selective frequency-reconfigurable antenna for cognitive radio applications," IEEE Antennas and Wireless Propagation Letters, vol. 13, pp. 515-518, 2014.

[28] R. N. Simon, Coplanar Waveguide Circuits Components and Systems, John Wiley \& Sons, New York, NY, USA, 2001.

[29] C. A. Balanis, Antenna Theory Analysis and Design, John Wiley \& Sons, Hoboken, NJ, USA, 2005.

[30] BAR88-02 Series Infineon PIN Diode Datasheet, http://www .infineon.com.

[31] BB145 NXP Semiconductors Varactor diode Datasheet, http:// www.nxp.com.

[32] H. A. Wheeler, "The radiansphere around a small antenna," Proceedings of the IRE, vol. 47, no. 8, pp. 1325-1331, 1959.

[33] E. H. Newman, P. Bohley, and C. H. Walter, "Two methods for the measurement of antenna efficiency," IEEE Transactions on Antennas and Propagation, vol. 23, no. 4, pp. 457-461, 1975. 


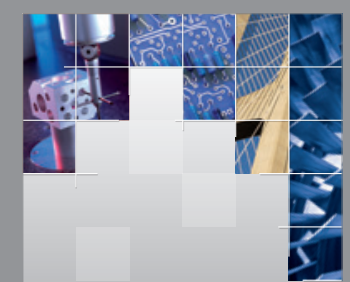

\section{Enfincering}
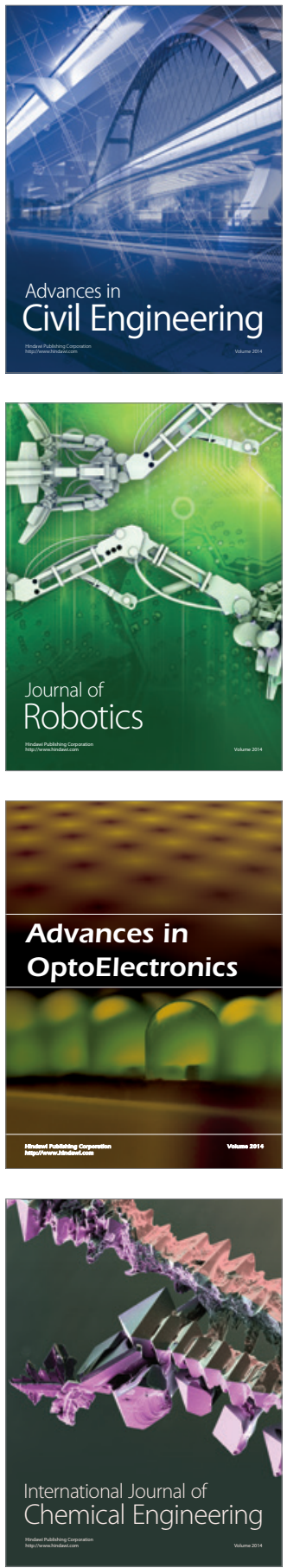

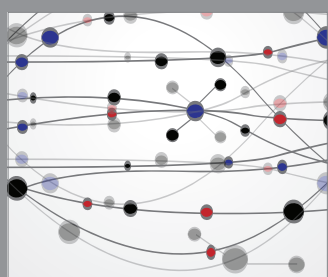

The Scientific World Journal

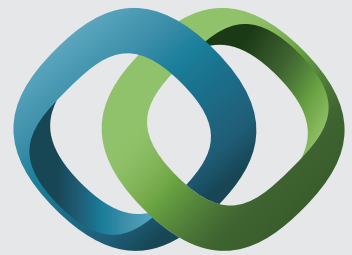

\section{Hindawi}

Submit your manuscripts at

http://www.hindawi.com
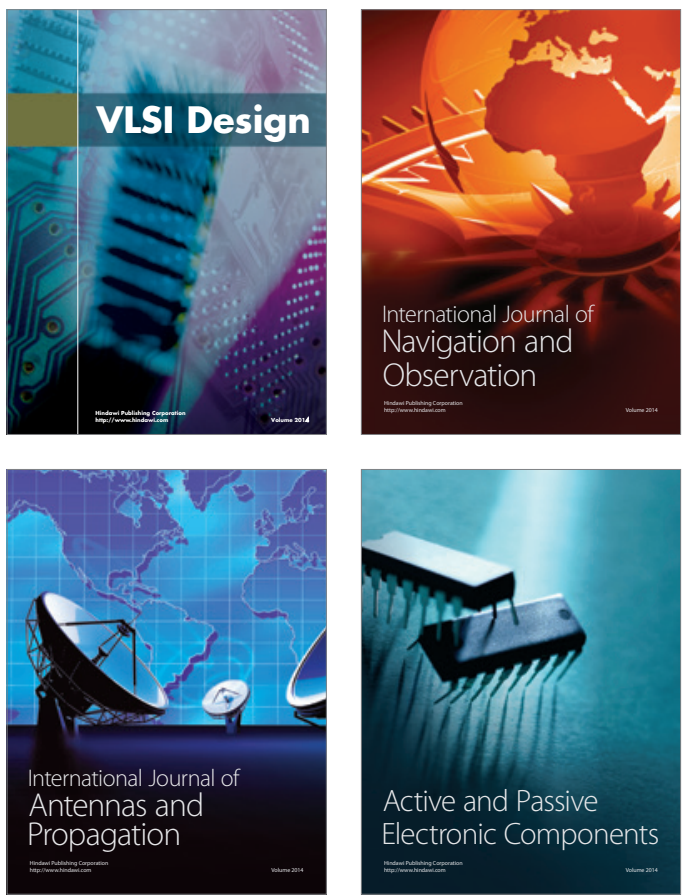
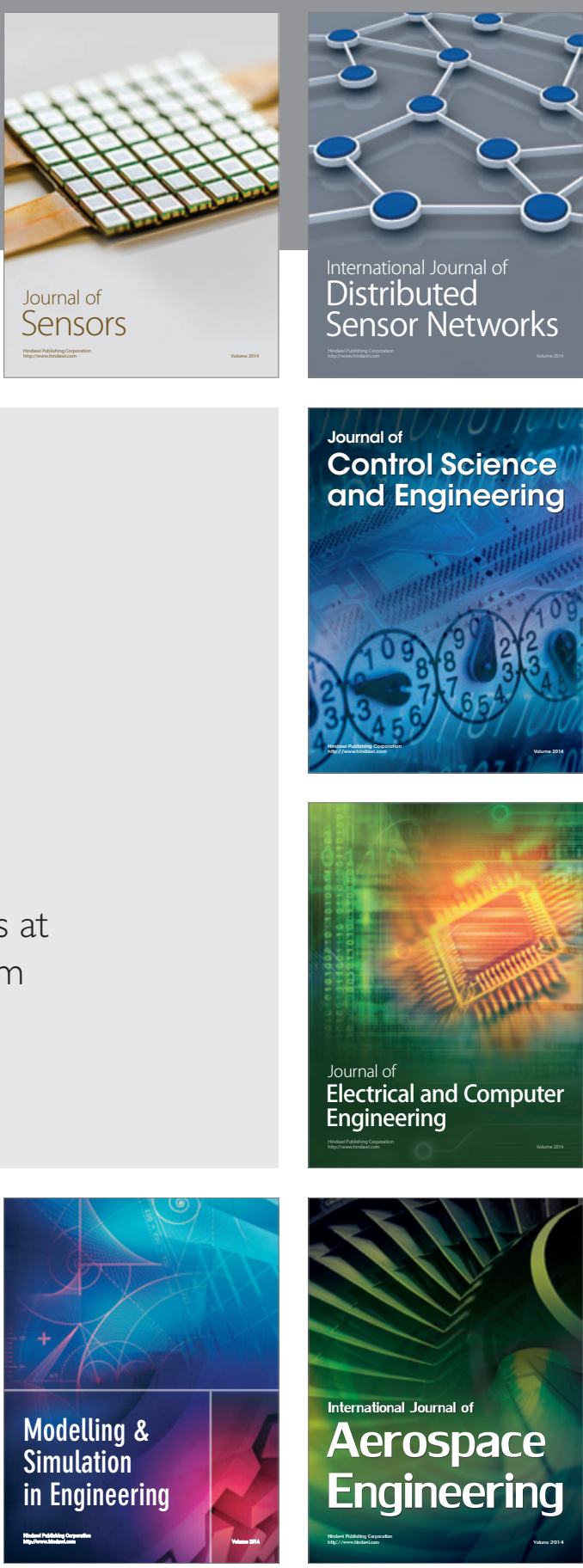

International Journal of

Distributed

Sensor Networks

Journal of

Control Science

and Engineering
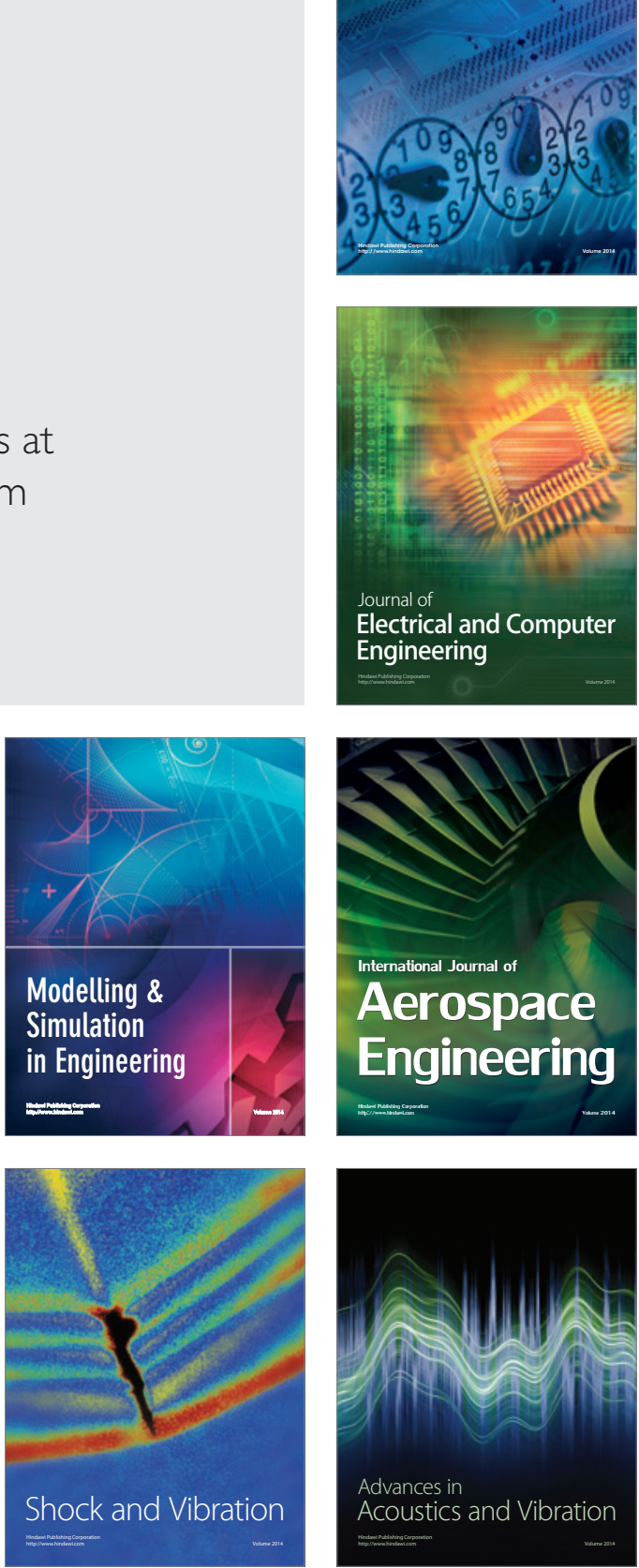